\title{
REGULARITY OF RENORMALIZED SELF-INTERSECTION LOCAL TIME FOR FRACTIONAL BROWNIAN MOTION*
}

\author{
YAOZHONG $\mathrm{HU}^{\dagger}$ AND DAVID NUALART ${ }^{\dagger}$
}

Abstract. Let $B_{t}^{H}$ be a $d$-dimensional fractional Brownian motion with Hurst parameter $H \in$ $(0,1)$. We study the regularity, in the sense of the Malliavin calculus, of the renormalized selfintersection local time

$$
\ell=\int_{0}^{T} \int_{0}^{t} \delta_{0}\left(B_{t}^{H}-B_{s}^{H}\right) d s d t-\mathbb{E}\left(\int_{0}^{T} \int_{0}^{t} \delta_{0}\left(B_{t}^{H}-B_{s}^{H}\right) d s d t\right)
$$

where $\delta_{0}$ is the Dirac delta function.

1. Introduction. The fractional Brownian motion on $\mathbb{R}^{d}$ with Hurst parameter $H \in(0,1)$ is a $d$-dimensional Gaussian process $B^{H}=\left\{B_{t}^{H}, t \geq 0\right\}$ with mean zero and covariance function given by

$$
\mathbb{E}\left(B_{t}^{H, i} B_{s}^{H, j}\right)=\frac{\delta_{i j}}{2}\left(t^{2 H}+s^{2 H}-|t-s|^{2 H}\right),
$$

where $i, j=1, \ldots, d$, and $s, t \geq 0$. We will assume that $d \geq 2$. The self-intersection local time of $B^{H}$ is formally defined by

$$
I=\int_{0}^{T} \int_{0}^{t} \delta_{0}\left(B_{t}^{H}-B_{s}^{H}\right) d s d t
$$

where $\delta_{0}(x)$ is the Dirac delta function. Using the heat kernel

$$
p_{\varepsilon}(x)=(2 \pi \varepsilon)^{-d / 2} e^{-\frac{|x|^{2}}{2 \varepsilon}}
$$

we approximate the self-intersection local time of $B^{H}$ by

$$
I_{\varepsilon}=\int_{0}^{T} \int_{0}^{t} p_{\varepsilon}\left(B_{t}^{H}-B_{s}^{H}\right) d s d t .
$$

The asymptotic behavior of $I_{\varepsilon}$ as $\varepsilon$ tends to zero is studied in [5], and the following results are proved.

i) If $H<\frac{1}{d}$, then $I_{\varepsilon}$ converges in $L^{2}$ as $\varepsilon$ tends to zero.

ii) If $\frac{1}{d}<H<\frac{3}{2 d}$, then

$$
I_{\varepsilon}-T C_{H, d} \varepsilon^{-\frac{d}{2}+\frac{1}{2 H}}
$$

converges in $L^{2}$ as $\varepsilon$ tends to zero to a limit $\ell$, where

$$
C_{H, d}=\frac{1}{(2 \pi)^{\frac{d}{2}}} \int_{0}^{\infty}\left(z^{\frac{1}{2 H}}+1\right)^{-\frac{d}{2}} d z
$$

\footnotetext{
${ }^{*} \mathrm{Y} . \mathrm{Hu}$ is supported in part by the National Science Foundation under Grant No. DMS0504783; D. Nualart is supported in part by the National Science Foundation under Grant No. DMS0604207.

${ }^{\dagger}$ Department of Mathematics, University of Kansas, 405 Snow Hall, Lawrence, Kansas 66045-2142. E-mail: hu@math.ku.edu
} 
iii) If $\frac{1}{d}=H<\frac{3}{2 d}$, then

$$
I_{\varepsilon}-\frac{T}{2 H(2 \pi)^{\frac{d}{2}}} \log \frac{1}{\varepsilon}
$$

converges in $L^{2}$ as $\varepsilon$ tends to zero.

iv) If $\frac{3}{2 d} \leq H<\frac{3}{4}$, then the random variables

$$
\left\{\begin{array}{lll}
\frac{1}{\left(\log \frac{1}{\varepsilon}\right)^{\frac{1}{2}}}\left(I_{\varepsilon}-\mathbb{E}\left(I_{\varepsilon}\right)\right) & \text { if } & H=\frac{3}{2 d} \\
\varepsilon^{\frac{d}{2}-\frac{3}{4 H}}\left(I_{\varepsilon}-\mathbb{E}\left(I_{\varepsilon}\right)\right) & \text { if } & H>\frac{3}{2 d}
\end{array}\right.
$$

converge as $\varepsilon$ tends to zero in distribution to a normal law $N\left(0, T \sigma^{2}\right)$, where $\sigma^{2}$ is a constant depending on $d$ and $H$.

We denote by $\ell$ the limit introduced in ii) and iii). It turns out that $\ell$ is also equal to the limit in $L^{2}$ of $I_{\varepsilon}-\mathbb{E}\left(I_{\varepsilon}\right)$ as $\varepsilon$ tends to zero. If $H<\frac{1}{d}$, then $\ell$ will be defined as the limit in $L^{2}$ of $I_{\varepsilon}-\mathbb{E}\left(I_{\varepsilon}\right)$ as $\varepsilon$ tends to zero. The random variable $\ell$ is called the renormalized self-intersection local time of the fractional Brownian motion.

In this paper we shall study the regularity, in the sense of Malliavin calculus, of the renormalized self-intersection local time $\ell$, assuming $H<\frac{3}{2 d}$. We prove that, for any real $\alpha>0, \ell$ belongs to the Sobolev space $\mathbb{D}^{\alpha, 2}$, provided $H<\min \left(\frac{3}{2 d}, \frac{2(\alpha \wedge 1)}{d+2 \alpha}\right)$. This result generalizes that obtained by $\mathrm{Hu}$ in [4] in the case $\alpha=1$. The proof of this result is established via chaos expansions.

In Section 2, we recall the chaos expansion of self-intersection local time obtained in [5]. In Section 3, we state and prove the main result of the paper.

2. Wiener chaos expansion of the self-intersection local time. In this section we recall the Wiener chaos expansion of the renormalized self-intersection local time $\ell$ obtained in [5].

Let $\mathcal{H}$ be the Hilbert space defined as the closure of set $\mathcal{E}$ of step functions from $\mathbb{R}_{+}$to $\mathbb{R}^{d}$ with respect to the scalar product

$$
\left\langle\left(\mathbf{1}_{\left[0, t_{1}\right]}, \ldots, \mathbf{1}_{\left[0, t_{d}\right]}\right),\left(\mathbf{1}_{\left[0, s_{1}\right]}, \ldots, \mathbf{1}_{\left[0, s_{d}\right]}\right)\right\rangle_{\mathcal{H}}=\frac{1}{2^{d}} \prod_{i=1}^{d}\left(t_{i}^{2 H}+s_{i}^{2 H}-\left|t_{i}-s_{i}\right|^{2 H}\right) .
$$

Then, the mapping $\mathbf{1}_{[0, t]} \rightarrow B_{t}^{H}$ is a linear isometry between $\mathcal{H}$ and the Gaussian space spanned by $B^{H}$. For any $n \geq 1$ we denote by $I_{n}$ the multiple stochastic integral which provides an isometry between the symmetric tensor product $(\mathcal{H})^{\otimes n}$ equipped with the norm $\sqrt{n !}\|\cdot\|_{\mathcal{H}_{\otimes n}}$ and the $n$th Wiener chaos of $B^{H}$.

Given a multi-index $\mathbf{i}_{n}=\left(i_{1}, \ldots, i_{n}\right), 1 \leq i_{j} \leq d$, we set

$$
\alpha\left(\mathbf{i}_{n}\right)=\mathbb{E}\left[X_{i_{1}} \cdots X_{i_{n}}\right]
$$


where the $X_{i}$ are independent $N(0,1)$ random variables. Notice that

$$
\alpha\left(\mathbf{i}_{2 m}\right)=\frac{\left(2 m_{1}\right) ! \cdots\left(2 m_{d}\right) !}{\left(m_{1}\right) ! \cdots\left(m_{d}\right) ! 2^{m}},
$$

if $n=2 m$ is even and for each $k=1, \ldots, d$, the number of components of $\mathbf{i}_{2 m}$ equal to $k$, denoted by $2 m_{k}$, is also even, and $\alpha\left(\mathbf{i}_{n}\right)=0$, otherwise.

Proposition 1. Assume $H d<\frac{3}{2}$. Then, we have

$$
\ell=\sum_{m=1}^{\infty} I_{2 m}\left(f_{2 m}\right)
$$

where $f_{2 m}$ is the element of $(\mathcal{H})^{\otimes 2 m}$ given by

$$
\begin{aligned}
f_{2 m}\left(\mathbf{i}_{2 m}, r_{1}, \ldots, r_{2 m}\right)= & \frac{(2 \pi)^{-\frac{d}{2}} \alpha\left(\mathbf{i}_{2 m}\right)}{(2 m) !} \\
& \times \int_{0}^{T} \int_{0}^{t} d s d t|t-s|^{-H d-2 H m} \prod_{j=1}^{2 m} \mathbf{1}_{[s, t]}\left(r_{j}\right) .
\end{aligned}
$$

Let us introduce the following notation.

$$
\lambda=|t-s|^{2 H}, \quad \rho=\left|t^{\prime}-s^{\prime}\right|^{2 H},
$$

and

$$
\mu=\frac{1}{2}\left[\left|s-t^{\prime}\right|^{2 H}+\left|s^{\prime}-t\right|^{2 H}-\left|t-t^{\prime}\right|^{2 H}-\left|s-s^{\prime}\right|^{2 H}\right] .
$$

Notice that $\lambda$ is the variance of $B_{t}^{H, 1}-B_{s}^{H, 1}, \rho$ is the variance of $B_{t^{\prime}}^{H, 1}-B_{s^{\prime}}^{H, 1}$, and $\mu$ is the covariance between $B_{t}^{H, 1}-B_{s}^{H, 1}$ and $B_{t^{\prime}}^{H, 1}-B_{s^{\prime}}^{H, 1}$, where $B^{H, 1}$ denotes a one-dimensional fractional Brownian motion with Hurst parameter $H$.

The $L^{2}$-norm of the $2 m$ th Wiener chaos of $\ell$ can be computed as follows.

$$
\begin{aligned}
\mathbb{E}\left[\left(I_{2 m}\left(f_{2 m}\right)\right)^{2}\right]= & (2 m) !\left\|f_{2 m}\right\|_{\mathcal{H}^{\otimes(2 m)}}^{2} \\
= & (2 m) ! \sum_{m_{1}+\cdots+m_{d}=m} \frac{(2 m) !}{\left(2 m_{1}\right) ! \cdots\left(2 m_{d}\right) !} \frac{(2 \pi)^{-d}}{((2 m) !)^{2}} \alpha\left(\mathbf{i}_{2 m}\right)^{2} \\
& \times \int_{\mathcal{T}} \lambda^{-\frac{d}{2}-m} \rho^{-\frac{d}{2}-m} \mu^{2 m} d s d t d s^{\prime} d t^{\prime} \\
= & \frac{\alpha_{m}}{(2 \pi)^{d} 2^{2 m}} \int_{\mathcal{T}}(\lambda \rho)^{-\frac{d}{2}-m} \mu^{2 m} d s d t d s^{\prime} d t^{\prime},
\end{aligned}
$$

where

$$
\alpha_{m}=\sum_{m_{1}+\cdots+m_{d}=m} \frac{\left(2 m_{1}\right) ! \cdots\left(2 m_{d}\right) !}{\left(m_{1} !\right)^{2} \cdots\left(m_{d} !\right)^{2}}
$$


and

$$
\mathcal{T}=\left\{\left(s, t, s^{\prime}, t^{\prime}\right): 0<s<t<T, 0<s^{\prime}<t^{\prime}<T\right\} .
$$

The following lemma will be useful later.

Lemma 2. For any $z \in[0,1)$ we have

$$
\sum_{m=0}^{\infty} \frac{(2 m) !}{(m !)^{2} 2^{2 m}} z^{m}=\frac{1}{\sqrt{1-z}}
$$

Proof. This is a well-known result that can be checked, for instance, by noticing that

$$
\sum_{m=0}^{\infty} \frac{(2 m) !}{(m !)^{2} 2^{2 m}} z^{m}=\sqrt{2 \pi} \mathbb{E}\left(e^{z(Y / 2)^{2}}\right),
$$

where $Y$ is a standard normal random variable.

3. Regularity of the renormalized self-intersection local time. For any $\alpha>0$, we denote by $\mathbb{D}^{\alpha, 2}$ the class of "smooth" functionals of the fractional Brownian motion, in the sense of Meyer-Watanabe. That is,

$$
\mathbb{D}^{\alpha, 2}=\left\{F \in L^{2}: \sum_{n=0}^{\infty}(n+1)^{\alpha} \mathbb{E}\left(\left(J_{n}(F)\right)^{2}\right)<\infty\right\},
$$

where $J_{n}(F)$ is the $n$-th chaos of $F$, namely, $F=\sum_{n=0}^{\infty} J_{n}(F)$.

The following theorem is the main result of this paper.

Theorem 3. Fix $\alpha>0$. Assume that $H<\min \left(\frac{3}{2 d}, \frac{2(\alpha \wedge 1)}{d+2 \alpha}\right)$. Then the renormalized self-intersection local time $\ell$ belongs to $\mathbb{D}^{\alpha, 2}$.

REMARK 4. If $\alpha=1$, we recover the result by $\mathrm{Hu}$ [4].

The theorem is the direct consequence of the following two lemmas which are themselves interesting.

Lemma 5. a) The renormalized self-intersection local time $\ell$ belongs to $\mathbb{D}^{N, 2}$, where $N \geq 1$, is an integer, if and only if

$$
\int_{\mathcal{T}} \mu^{2 N} \delta^{-\frac{d}{2}-N} d s d t d s^{\prime} d t^{\prime}<\infty
$$

b) The renormalized self-intersection local time $\ell$ belongs to $\mathbb{D}^{N+\beta, 2}$, where $N \geq 0$, is an integer, and $0<\beta<1$, if for some $1>\beta^{\prime}>\beta$

$$
\int_{\mathcal{T}} \mu^{2\left(N+\beta^{\prime}\right)} \delta^{-\frac{d}{2}-N-\beta^{\prime}} d s d t d s^{\prime} d t^{\prime}<\infty .
$$

Proof. From (6) we obtain that, for all $\alpha>0$, a necessary and sufficient condition for $\ell$ to be in $\mathbb{D}^{\alpha, 2}$ is

$$
B:=\sum_{m=1}^{\infty} \frac{m^{\alpha} \alpha_{m}}{2^{2 m}} \int_{\mathcal{T}} \frac{\gamma^{m}}{(\lambda \rho)^{\frac{d}{2}}} d s d t d s^{\prime} d t^{\prime}<\infty,
$$


where

$$
\gamma=\frac{\mu^{2}}{\lambda \rho}
$$

Using Lemma 2 we deduce the following formula for all $z \in[0,1)$

$$
\sum_{m=0}^{\infty} \frac{\alpha_{m}}{2^{2 m}} z^{m}=(1-z)^{-\frac{d}{2}}
$$

Suppose first that $\alpha=N$ is an integer. In this case, differentiating both sides of (9) $N$ times with respect to $z$ yields

$$
\sum_{m=N}^{\infty} \frac{\alpha_{m}}{2^{2 m}} m(m-1) \cdots(m-N+1) z^{m-N}=C(1-z)^{-\frac{d}{2}-N},
$$

where $C=\frac{d}{2}\left(\frac{d}{2}+1\right) \cdots\left(\frac{d}{2}+N-1\right)$. Hence,

$$
\sum_{m=N}^{\infty} \frac{\alpha_{m}}{2^{2 m}} m(m-1) \cdots(m-N+1) z^{m}=C z^{N}(1-z)^{-\frac{d}{2}-N},
$$

and we get that (8) is equivalent to

$$
\int_{\mathcal{T}} \frac{\gamma^{N}(1-\gamma)^{-\frac{d}{2}-N}}{(\lambda \rho)^{\frac{d}{2}}} d s d t d s^{\prime} d t^{\prime}=\int_{\mathcal{T}} \mu^{2 N} \delta^{-\frac{d}{2}-N} d s d t d s^{\prime} d t^{\prime}<\infty,
$$

where

$$
\delta=\lambda \rho-\mu^{2} .
$$

This proves part a) of the lemma.

Suppose now that $k=N+\beta$, with $0<\beta<1$, and $N \geq 0$. Multiplying both members of Equation (9) by $(y-z)^{-\beta}$ and integrating in the variable $z$ from 0 to $y$, we obtain

$$
\sum_{m=0}^{\infty} \frac{\alpha_{m}}{2^{2 m}} \frac{\Gamma(1-\beta) \Gamma(m)}{\Gamma(1-\beta+m)} y^{m-\beta+1}=\int_{0}^{y}(1-z)^{-\frac{d}{2}}(y-z)^{-\beta} d z .
$$

Hence,

$$
\sum_{m=0}^{\infty} \frac{\alpha_{m}}{2^{2 m}} \frac{\Gamma(1-\beta) \Gamma(m)}{\Gamma(1-\beta+m)} y^{m}=\int_{0}^{1}(1-y t)^{-\frac{d}{2}}(1-t)^{-\beta} d t .
$$

Differentiating this identity $N+1$ times with respect to $z$ yields

$$
\begin{aligned}
& \sum_{m=N+1}^{\infty} \frac{\alpha_{m}}{2^{2 m}} m(m-1) \cdots(m-N-2) \frac{\Gamma(1-\beta) \Gamma(m)}{\Gamma(1-\beta+m)} z^{m-N-1} \\
= & C \int_{0}^{1}(1-z t)^{-\frac{d}{2}-N-1} t^{N+1}(1-t)^{-\beta} d t,
\end{aligned}
$$


where $C=\frac{d}{2}\left(\frac{d}{2}+1\right) \cdots\left(\frac{d}{2}+N\right)$. Hence, (8) is equivalent to

$$
\int_{\mathcal{T}}(\lambda \rho)^{-\frac{d}{2}} \gamma^{N+1}\left(\int_{0}^{1}(1-\gamma y)^{-\frac{d}{2}-N-1} y^{N+1}(1-y)^{-\beta} d y\right) d s d t d s^{\prime} d t^{\prime}<\infty .
$$

We claim that for all $\beta^{\prime}>\beta$,

$$
\int_{0}^{1}(1-y)^{-\beta}(1-\gamma y)^{-\frac{d}{2}-N-1} d y \leq k(1-\gamma)^{-\frac{d}{2}-N-\beta^{\prime}}
$$

In fact, $(1-\gamma y)^{-\frac{d}{2}-N-1} \leq(1-y)^{\beta^{\prime}-1}(1-\gamma)^{-\frac{d}{2}-N-\beta^{\prime}}$. Thus,

$$
\begin{aligned}
\int_{0}^{1}(1-y)^{-\beta}(1-\gamma y)^{-\frac{d}{2}-N-1} d y & \leq(1-\gamma)^{-\frac{d}{2}-N-\beta^{\prime}} \int_{0}^{1}(1-y)^{-\beta+\beta^{\prime}-1} d y \\
& \leq \frac{1}{\beta^{\prime}-\beta}(1-\gamma)^{-\frac{d}{2}-N-\beta^{\prime}} .
\end{aligned}
$$

Hence, (10) holds if

$$
\begin{aligned}
& \int_{\mathcal{T}}(\lambda \rho)^{-\frac{d}{2}} \gamma^{N+1}(1-\gamma)^{-\frac{d}{2}-N-\beta^{\prime}} d s d t d s^{\prime} d t^{\prime} \\
= & \int_{\mathcal{T}}(\lambda \rho)^{\beta^{\prime}-1} \mu^{2(N+1)} \delta^{-\frac{d}{2}-N-\beta^{\prime}} d s d t d s^{\prime} d t^{\prime}<\infty,
\end{aligned}
$$

and (7) holds because $\mu^{2} \leq \lambda \rho$.

LEMma 6. Fix a positive real number $\alpha>0$. Suppose that $H<\min \left(\frac{3}{2 d}, \frac{2(\alpha \wedge 1)}{d+2 \alpha}\right)$. Then

$$
\int_{\mathcal{T}} \mu^{2 \alpha} \delta^{-\frac{d}{2}-\alpha} d s d t d s^{\prime} d t^{\prime}<\infty
$$

Proof. Denote

$$
\mathcal{T} \cap\left\{s<s^{\prime}\right\}=\mathcal{T}_{1} \cup \mathcal{T}_{2} \cup \mathcal{T}_{3},
$$

where

$$
\begin{aligned}
& \mathcal{T}_{1}=\left\{\left(t, s, t^{\prime}, s^{\prime}\right): 0<s<s^{\prime}<t<t^{\prime}<T\right\}, \\
& \mathcal{T}_{2}=\left\{\left(t, s, t^{\prime}, s^{\prime}\right): 0<s<s^{\prime}<t^{\prime}<t<T\right\}, \\
& \mathcal{T}_{3}=\left\{\left(t, s, t^{\prime}, s^{\prime}\right): 0<s<t<s^{\prime}<t^{\prime}<T\right\} .
\end{aligned}
$$

We will make use of the notation:

i) If $\left(t, s, t^{\prime}, s^{\prime}\right) \in \mathcal{T}_{1}$, we put $a=s^{\prime}-s, b=t-s^{\prime}$ and $c=t^{\prime}-t$. On this region, the functions $\lambda, \rho$ and $\mu$ defined in (4) and (5) take the following values

$$
\begin{aligned}
& \lambda=\lambda_{1}:=\lambda_{1}(a, b, c):=(a+b)^{2 H}, \rho=\rho_{1}:=(b+c)^{2 H}, \\
& \mu=\mu_{1}:=\mu_{1}(a, b, c):=\frac{1}{2}\left[(a+b+c)^{2 H}+b^{2 H}-c^{2 H}-a^{2 H}\right] .
\end{aligned}
$$


ii) If $\left(t, s, t^{\prime}, s^{\prime}\right) \in \mathcal{T}_{2}$, we put $a=s^{\prime}-s, b=t^{\prime}-s^{\prime}$ and $c=t-t^{\prime}$. On this region we will have

$$
\begin{aligned}
& \lambda=\lambda_{2}:=b^{2 H}, \rho=\rho_{2}:=(a+b+c)^{2 H}, \\
& \mu=\mu_{2}:=\frac{1}{2}\left[(b+c)^{2 H}+(a+b)^{2 H}-c^{2 H}-a^{2 H}\right] .
\end{aligned}
$$

iii) If $\left(t, s, t^{\prime}, s^{\prime}\right) \in \mathcal{T}_{3}$, we put $a=t-s, b=s^{\prime}-t$ and $c=t^{\prime}-s^{\prime}$. On this region we will have

$$
\begin{aligned}
& \lambda=\lambda_{3}:=a^{2 H}, \rho=\rho_{3}:=c^{2 H}, \\
& \mu=\mu_{3}:=\frac{1}{2}\left[(a+b+c)^{2 H}+b^{2 H}-(b+c)^{2 H}-(a+b)^{2 H}\right] .
\end{aligned}
$$

For $i=1,2,3$ we set

$$
\delta_{i}=\lambda_{i} \rho_{i}-\mu_{i}^{2}
$$

Note that $\lambda_{i}, \rho_{i}, \mu_{i}$ and so on, $i=1,2,3$, are functions of $a, b$, and $c$.

In the sequel we will denote by $k$ a generic constant that may depend on $H$ and $d$.

The following lower bounds were obtained by $\mathrm{Hu}$ in [4] using the local nondeterminism property of the fractional Brownian motion (see Berman [2]).

$$
\begin{aligned}
& \delta_{1} \geq k\left[(a+b)^{2 H} c^{2 H}+(b+c)^{2 H} a^{2 H}\right], \\
& \delta_{i} \geq k \lambda_{i} \rho_{i}, i=2,3 .
\end{aligned}
$$

Using the above decomposition of the region $\mathcal{T}$, it suffices to show that $A_{i}<\infty$, for $i=1,2,3$, where

$$
A_{i}:=\int_{[0, T]^{3}} \mu_{i}^{2 N} \delta_{i}^{-\frac{d}{2}-N} d a d b d c .
$$

Then the proof of the lemma will de done in three steps:

Step 1. We claim that

$$
A_{1}<\infty
$$

We have

$$
\begin{aligned}
\mu_{1} & =\frac{1}{2}\left((a+b+c)^{2 H}+b^{2 H}-a^{2 H}-c^{2 H}\right) \\
& =\frac{1}{2}\left(\left(a^{2}+b^{2}+c^{2}+2 a b+2 a c+2 b c\right)^{H}+b^{2 H}-a^{2 H}-c^{2 H}\right) \\
& \leq b^{2 H}+2^{H-1} a^{H} b^{H}+2^{H-1} a^{H} c^{H}+2^{H-1} b^{H} c^{H} .
\end{aligned}
$$


The, using (18) yields

$$
\begin{aligned}
\mu_{1}^{2 \alpha} & \leq k\left(b^{4 \alpha H}+\left(a^{2 \alpha H} b^{2 \alpha H}+a^{2 \alpha H} c^{2 \alpha H}+b^{2 \alpha H} c^{2 \alpha H}\right)\right) \\
& \leq 3 k\left(b^{2 \alpha H}+\delta^{\alpha}\right) .
\end{aligned}
$$

As a consequence,

$$
\mu_{1}^{2 \alpha} \delta_{1}^{-\frac{d}{2}-\alpha} \leq k\left(\delta_{1}^{-\frac{d}{2}}+b^{4 \alpha H} \delta_{1}^{-\frac{d}{2}-\alpha}\right) .
$$

Using again (18) we obtain

$$
\begin{aligned}
\delta_{1}^{-\frac{d}{2}} & \leq k\left[(a+b)^{H}(b+c)^{H} a^{H} c^{H}\right]^{-\frac{d}{2}} \\
& \leq k(a b c)^{-\frac{2}{3} H d}
\end{aligned}
$$

where $-\frac{2}{3} H d>-1$.

In order to treat the second term of (20) we consider two different cases. Assume first that $d \leq 6 \alpha$. Then

$$
\begin{aligned}
b^{2 \alpha H} \delta_{1}^{-\frac{d}{2}-\alpha} & \leq k\left[(a+b)^{2 H} c^{2 H}+(b+c)^{2 H} a^{2 H}\right]^{-\frac{d}{2}-\alpha} b^{4 \alpha H} \\
& \leq k\left[(b c)^{2 H}+(b a)^{2 H}\right]^{-\frac{d}{2}-\alpha} b^{4 \alpha H} \\
& \leq k(a c)^{-H\left(\frac{d}{2}+\alpha\right)} b^{H(2 \alpha-d)}
\end{aligned}
$$

and both exponents are larger than -1 , because $H<\frac{2}{d+2 \alpha} \leq \frac{1}{d-2 \alpha}$.

For $d>6 \alpha$, we make use of the estimate

$$
\begin{aligned}
b^{2 \alpha H} \delta_{1}^{-\frac{d}{2}-\alpha} & \leq k\left[(a+b)^{H} c^{H}(b+c)^{H} a^{H}\right]^{-\frac{d}{2}-\alpha} b^{4 \alpha H} \\
& \leq k(a c)^{-\left(\beta_{1}+1\right)\left(\frac{d}{2}+\alpha\right) H} b^{4 \alpha H-\beta_{2}(d+2 \alpha) H}
\end{aligned}
$$

where $\beta_{1}, \beta_{2} \geq 0$, and $\beta_{1}+\beta_{2}=1$. Taking

$$
\beta_{1}=\frac{d-6 \alpha}{3(d+2 \alpha)}, \beta_{2}=\frac{2 d+12 \alpha}{3(d+2 \alpha)}
$$

we obtain

$$
b^{2 \alpha H} \delta_{2}^{-\frac{d}{2}-\alpha} \leq k(a b c)^{-\frac{2 d H}{3}} .
$$

Step 2. We claim that

$$
A_{2}<\infty \text {. }
$$

If $H \geq \frac{1}{2}$ we have

$$
\begin{aligned}
\mu_{2} & =\frac{1}{2}\left((b+c)^{2 H}+(a+b)^{2 H}-a^{2 H}-c^{2 H}\right) \\
& =H b \int_{0}^{1}\left[(a+b u)^{2 H-1}+(c+b u)^{2 H-1}\right] d u \\
& \leq k b(a+b+c)^{2 H-1} .
\end{aligned}
$$


Therefore, using (19)

$$
\mu_{2}^{2 \alpha} \delta_{2}^{-\frac{d}{2}-\alpha} \leq b^{-H(d+2 \alpha)+2 \alpha}(a+b+c)^{H(2 \alpha-d)-2 \alpha} .
$$

Using the inequality $a+b+c \geq C a^{\beta} c^{\beta} b^{1-2 \beta}$, with $\beta=\frac{2 H d}{3 H d+6 \alpha-6 \alpha H}$, we obtain

$$
\mu_{2}^{2 \alpha} \delta_{2}^{-\frac{d}{2}-\alpha} \leq k(a b c)^{-\frac{2 d H}{3}}
$$

Notice that $\beta \in\left(0, \frac{1}{2}\right]$, because $H<\frac{2 \alpha}{d+2 \alpha}$.

Suppose now that $H<\frac{1}{2}$. In this case we have

$$
\mu_{2} \leq k b\left(a^{\beta(2 H-1)} b^{(1-\beta)(2 H-1)}+c^{\beta(2 H-1)} b^{(1-\beta)(2 H-1)}\right),
$$

for all $\beta \in[0,1]$. Hence,

$$
\begin{aligned}
\mu_{2}^{2 \alpha} \delta_{2}^{-\frac{d}{2}-\alpha} \leq & k a^{\beta(2 H-1) 2 \alpha} b^{(1-\beta)(2 H-1) 2 \alpha+2 \alpha} \delta_{2}^{-\frac{d}{2}-\alpha} \\
& +k c^{\beta(2 H-1) 2 \alpha} b^{(1-\beta)(2 H-1) 2 \alpha+2 \alpha} \delta_{2}^{-\frac{d}{2}-\alpha} \\
= & I_{1}+I_{2} .
\end{aligned}
$$

By symmetry it suffices to treat the term $I_{1}$. We have

$$
I_{1} \leq k a^{\beta(2 H-1) 2 \alpha} b^{2(1-2 \beta) \alpha H+2 \alpha \beta-d H}(a+b+c)^{-d H-2 \alpha H} .
$$

Now we make use of the lower bound

$$
(a+b+c)^{-1} \geq k a^{\gamma_{1}} b^{\gamma_{2}} c^{\gamma_{3}},
$$

where $\gamma_{1}+\gamma_{2}+\gamma_{3}=1$, and $\gamma_{1}, \gamma_{2}, \gamma_{3} \geq 0$. In this way we obtain

$$
I_{1} \leq k a^{\beta_{1}} b^{\beta_{2}} c^{\beta_{3}}
$$

where

$$
\begin{aligned}
& \beta_{1}=\beta(2 H-1) 2 \alpha-\gamma_{1} H(d+2 \alpha) \\
& \beta_{2}=2(1-2 \beta) \alpha H+2 \alpha \beta-d H-\gamma_{2} H(d+2 \alpha) \\
& \beta_{3}=-\gamma_{3} H(d+2 \alpha) .
\end{aligned}
$$

If $d \leq 6 \alpha$, we choose $\beta=0, \gamma_{1}=\gamma_{3}=\frac{1}{2}$, and $\gamma_{2}=0$, and we obtain the exponents

$$
\begin{aligned}
& \beta_{1}=\beta_{3}=-\frac{H(d+2 \alpha)}{2}>-1 \\
& \beta_{2}=H(2 \alpha-d)>-1
\end{aligned}
$$

If $d>6 \alpha$, we choose

$$
\beta=\frac{H(d-6 \alpha)}{6(1-2 H) \alpha}, \gamma_{1}=\frac{d+6 \alpha}{3(d+2 \alpha)}, \gamma_{2}=0, \gamma_{3}=\frac{2 d}{3(d+2 \alpha)},
$$


and we obtain the exponents

$$
\beta_{1}=\beta_{2}=\beta_{3}=-\frac{2 d H}{3}>-1 .
$$

Step 3.- We claim that

$$
A_{3}<\infty \text {. }
$$

In this case, (17) and the inequality

$$
b+v c+u a \geq k(v c u a)^{\beta} b^{1-2 \beta},
$$

with $\beta \in[0,1]$, yield

$$
\mu_{3} \leq k(a c)^{1+\beta(2 H-2)} b^{(1-2 \beta)(2 H-2)},
$$

provided $\beta<\frac{1}{2(1-H)}$. As a consequence,

$$
\mu_{3}^{2 \alpha} \delta_{3}^{-\frac{d}{2}-\alpha} \leq k(a c)^{[1+\beta(2 H-2)] 2 \alpha-d H-2 H \alpha} b^{(1-2 \beta)(2 H-2) 2 \alpha} .
$$

Choosing $\beta=\frac{6 \alpha-6 H \alpha-H d}{12 \alpha(1-H)}$, we obtain

$$
\mu_{3}^{2 \alpha} \delta_{3}^{-\frac{d}{2}-\alpha} \leq k(a c)^{-\frac{2 d H}{3}} .
$$

Notice that $\beta>0$ because $H<\frac{2 \alpha}{2 \alpha+d}<\frac{6 \alpha}{6 \alpha+d}$, and also $\beta<\frac{1}{2(1-H)}$.

\section{REFERENCES}

[1] S. Albeverio, Y. Z. Hu, And X. Y. Zhou, A remark on non smoothness of self intersection local time of planar Brownian motion, Statist. Probab. Letters, 32(1997), pp. 57-65.

[2] S. M. Berman, Local nondeterminism and local times of Gaussian processes, Bull. Amer. Math. Soc. $79(1973)$, pp. $475-477$.

[3] Y. Z. Hu, On the self-intersection local time of Brownian motion - via chaos expansion, Publicacions Matemàtiques, 40(1996), pp. 337-350.

[4] Y. Z. Hu, Self-intersection local time of fractional Brownian motions-via chaos expansion, J. Math. Kyoto Univ., 41(2001), pp. 233-250.

[5] Y. Hu And D. Nualart, Renormalized self-intersection local time for fractional Brownian motion, Ann. Probab., 33(2005), pp. 948-983.

[6] P. Imkeller, V. Pérez-Abreu, and J. Vives, Chaos expansions of double intersection local time of Brownian motion in $\mathbb{R}^{d}$ and renormalization, Stochastic Process. Appl., 56(1995), pp. 1-34.

[7] D. Nualart, The Malliavin calculus and related topics. Probability and its Applications. Springer-Verlag, New York, 1995. 